\title{
Estudio de las opiniones de los futuros maestros sobre el uso de los videojuegos como recurso didáctico a través de un análisis cualitativo
}

\author{
Luis Manuel Soto-Ardila, Lina Melo Niño, Ana Caballero, Ricardo Luengo \\ luismanuel@unex.es, lvmelo@unex.es, acabcar@unex.es,rluengo@unex.es \\ Departamento de Didáctica de las Ciencias Experimentales y Matemáticas de la Universidad de \\ Extremadura, España.
}

DOI: 10.17013/risti.33. 48-63

\begin{abstract}
Resumen: El presente artículo pretende analizar las opiniones que los estudiantes del Grado de Educación Primaria tienen con respecto a la utilización de los videojuegos como recurso didáctico. Para ello se desarrolla una investigación descriptiva mediante un análisis cualitativo de los textos obtenidos a través de un foro utilizando, para ello, el Software webQDA. La muestra empleada para este estudio es de 52 alumnos. El objetivo es conocer si los estudiantes se encuentran a favor o en contra del uso de los videojuegos en un ámbito educativo y cuáles son los motivos que les llevan a decantarse por una u otra opinión. Se concluye que la mayoría del alumnado está a favor de los videojuegos por su carácter motivador y lúdico por desarrollar el pensamiento lógico, crítico y la resolución de problemas.
\end{abstract}

Palabras-clave: videojuegos; educación; ventajas e inconvenientes; maestros en formación inicial; webQDA.

Study of the opinions of the students of the Primary Education Degree on the use of videogames as a didactic resource through a qualitative analysis

\begin{abstract}
This article aims to analyze the opinions that the students of the Primary Education Degree have regarding the use of video games as a teaching resource. For this, a descriptive investigation is developed through a qualitative analysis of the texts obtained through a forum using, for this, the webQDA Software. The sample used for this study is 52 students. The objective is to know if the students are in favor or against the use of videogames in an educational environment and which are the reasons that lead them to opt for one or the other opinion. It is concluded that the majority of students are in favor of videogames because of their motivating and playful nature for developing logical, critical thinking and problem solving.
\end{abstract}

Keywords: video games; education; advantages and disadvantages; teachers in training; webQDA. 


\section{Introducción}

Está comprobado por la bibliografía científica, y por nuestras propias vivencias, que las nuevas tecnologías están cada vez más presentes en nuestra vida. Hoy en día todos tenemos un teléfono móvil, Tablet o un ordenador en casa. Tal es el uso de estas tecnologías que, los niños, desde pequeños, saben utilizarlas (de ahí que sean denominados "Nativos Digitales"). Esto se debe a que los niños, a diferencia de épocas pasadas, han nacido en una sociedad en la que, como se ha dicho anteriormente, las tecnologías de la información y de la comunicación (en adelante TIC) están muy presentes y son muy importantes para ellos (Martinez-Piñeiro, Vila y Gewerc, 2018).

Normalmente, a los niños suele gustarles jugar, y este hecho se ha ido manteniendo a lo largo de la historia. Este es el motivo por el que cada vez se crean más juegos y se van adaptando a la situación social del momento. En nuestra historia moderna, esos juegos han evolucionado hasta digitalizarse y adaptarse a las TIC. Como consecuencia de esa adaptación surgen los videojuegos, que han sido definidos de diversas formas:

Frasca (2001), define los videojuegos como "todas aquellas formas de software de entretenimiento, en las que se usa cualquier plataforma electrónica, y los jugadores (uno o varios) se encuentran en un entorno físico o de red" (p. 4).

A su vez, Zyda (2005), considera que los videojuegos no son más que "una prueba mental, que se realiza en ordenadores y que sigue una serie de reglas, cuyo objetivo fundamental es la diversión del usuario" (p. 25).

En nuestro caso, teniendo en cuenta nuestro propio criterio y basándonos en las definiciones anteriormente descritas, consideramos que los videojuegos son aquellos juegos digitales (principalmente audiovisuales) que permiten que el jugador interactúe con un entorno virtual, en el que deberá seguir una serie de reglas y superar los distintos desafíos. Presentan objetivos y temáticas variadas.

Teniendo en cuenta esta definición, los videojuegos pasan a ser una herramienta flexible que, con un diseño adecuado, puede ser utilizada como recurso educativo. En el apartado "Marco Teórico" se detallan varias investigaciones en las que se aborda el tema de los videojuegos desde diferentes perspectivas: los videojuegos como recurso didáctico y los posibles beneficios y/o problemas que los videojuegos pueden provocar. De esta forma, se establece una base formal para esta investigación.

\section{Marco Teórico}

En el siguiente apartado se describen algunas de las investigaciones en las que los videojuegos han sido utilizados como una posible herramienta de trabajo; en ellas se detallan cuáles son los beneficios que estos recursos pueden tener en la enseñanza de ciertos contenidos didácticos o cuáles son los problemas que pueden ocasionar en el niño si no se realiza un uso responsable de los mismos. Del mismo modo, se analiza la literatura científica sobre cómo los videojuegos pueden influir en otros ámbitos relacionados con la educación, como puede ser el ámbito de la salud o las necesidades educativas de los alumnos. 


\subsection{Videojuegos en la educación: ¿beneficiosos o perjudiciales para el niño?}

Los videojuegos presentan un amplio abanico de posibilidades como recurso para la enseñanza de contenidos didácticos, convirtiéndose en unas herramientas muy útiles para enseñar (Soto-Ardila, 2015). Eguía, Contreras-Espinosa y Solano-Albajes (2013) realizan una reflexión acerca de las posibilidades que los videojuegos presentan en la educación; establecen relaciones entre la utilización de los mismos y el desarrollo constructivo de actitudes, conocimientos y habilidades.

Autores como Dondim, Edvinsson y Moretti (2004) consideran los videojuegos como herramientas útiles para desarrollar algunas habilidades sociales necesarias para que el niño interaccione con el entorno. Otros aspectos como las habilidades sociales, el rendimiento, o el aprendizaje motivado, se ven beneficiados con el uso de los videojuegos, tal y como afirman Rosas, Nussbaum, Cumsille, Marianov, Correa y Flores (2003) y Albarracín, Hernández y Gorgorió (2019).

Autores como Brustolin y Malta (2017), abordan el tema de la atención como un factor que se ve beneficiado si se utilizan los juegos como recurso didáctico, incrementándose así los estudios sobre la gamificación para observar mejoras del alumnado en el aprendizaje.

La competencia digital o la llamada "alfabetización digital” es algo que es muy necesario en casi todos los aspectos de nuestra vida, dado que se requiere cierto manejo de las nuevas tecnologías para el desarrollo de multitud de trabajos. Los videojuegos pueden convertirse en una buena herramienta que favorezca el desarrollo de dicha competencia (Aguilar, Rubio y Viñals, 2013; Alonso y Yuste, 2014; Muros, Aragón y Bustos, 2013).

La concentración también se ve beneficiada con el uso de los videojuegos, así como el pensamiento lógico y crítico, o la resolución de problemas y la planificación de estrategias (Kirriemuir y Mcfarlane, 2004; Higgins, 2001). En este apartado se pueden mencionar, además, autores como Torres-Toukoumidis, Romero-Rodríguez, Pérez-Rodriguez y Björl (2016), que defienden la utilización de los videojuegos de estrategias, dado que fomentan el desarrollo de dichos aspectos.

Existen otros estudios que avalan los efectos positivos que, la utilización de los videojuegos presenta sobre las inteligencias múltiples de los alumnos de primaria. Del Moral, Guzmán y Fernández (2014) explican cómo el uso de los videojuegos fomenta el desarrollo de la inteligencia lógico-matemática y la viso-espacial en las niñas y la inteligencia lingüística e interpersonal en los niños.

Eguía, Contreras-Espinosa y Solano-Albajes (2013) desarrollan en su estudio una clasificación de las distintas áreas del aprendizaje en la que los videojuegos son beneficiosos para su desarrollo. A continuación, se muestra una tabla en la que aparece recogida dicha clasificación:

Pero no solo existen estudios en los que se habla de todos los beneficios que los videojuegos pueden tener, sino que, también, existen otros en los que se habla de los problemas que un uso no controlado de estos juegos puede ocasionar en los niños. 


\begin{tabular}{ll}
\hline Áreas del aprendizaje & Beneficios de los videojuegos \\
\hline Desarrollo personal y social & $\begin{array}{l}\text { Proporciona interés y motivación. } \\
\text { - Mantiene la atención y la concentración. } \\
\text { • Puede trabajarse corno parte de un grupo y se pueden } \\
\text { compartir recursos. }\end{array}$ \\
\hline Conocimiento y comprensión del mundo & $\begin{array}{l}\text { - Conocer algunas cosas que pasan. } \\
\text { - Uso temprano del control del software. }\end{array}$ \\
\hline & $\begin{array}{l}\text { - Anima a los niños a explicar lo que está pasando en el } \\
\text { juego. }\end{array}$ \\
\hline Lenguaje y alfabetización & $\begin{array}{l}\text { Uso del discurso, de la palabra para organizar, } \\
\text { sentimientos y eventos. }\end{array}$ \\
\hline Desarrollo creativo & $\begin{array}{l}\text { Respuesta en formas muy variadas. } \\
\text { - Uso de la imaginación a partir del diseño gráfico, la } \\
\text { música, y la narrativa de las historias. }\end{array}$ \\
\hline Desarrollo físico & $\begin{array}{l}\text { Control de la motricidad a partir del uso del ratón en la } \\
\text { navegación y selección de objetos. }\end{array}$ \\
\hline
\end{tabular}

Tabla 1 - Clasificación de los beneficios de los videojuegos en distintas áreas del aprendizaje (Eguía, Contreras-Espinosa y Solano-Albajes, 2013)

Así, Ferguson (2010) explica cómo los videojuegos que tienen unas temáticas "no adecuadas" para el niño, pueden ocasionar algunas conductas agresivas o patológicas en aquellos niños que los juegan.

En la literatura científica, además, se pueden encontrar algunas investigaciones en las que se abordan temas como la adicción ocasionada por un uso abusivo de los videojuegos. Dichas investigaciones consideran que los niños les dedican muchas horas a jugar a videojuegos, dejando desatendidas otras actividades como puede ser estudiar o, incluso, socializarse con sus familiares e iguales. Sin embargo, otros estudios muestran como el niño suele utilizar el videojuego de forma excesiva únicamente en el momento en el que conoce dicho videojuego, pero, con el paso del tiempo, ese uso suele quedar reducido y normalizado (Padilla, Collazos, Gutiérrez y Medina, 2012).

Otro aspecto negativo de los videojuegos suele referirse a la socialización del niño, dado que se considera que los videojuegos provocan aislamiento. Esto se debe a que, como se le dedica mucho tiempo a jugar, se pierden las ganas de salir a la calle y relacionarse con otras personas. Sin embargo, en la literatura encontramos muchos autores que defienden que, cuanto más “jugador” es el niño, más sociable se vuelve (Colwel, Grady y Rhaiti, 1995; Estallo, 1995).

\subsection{Videojuegos en otros ámbitos de la vida}

Tras un breve desarrollo de los beneficios o problemas que los videojuegos pueden presentar en el ámbito educativo, se considera necesario el estudio de los mismos en ámbitos diferentes al de la educación, como puede ser, por ejemplo, el de la salud o las Necesidades Educativas Especiales (en adelante NEE). 
En las aulas existe una gran diversidad de niños, algunos de ellos con ciertas dificultades que impiden un desarrollo educativo adecuado: niños con problemas en la motricidad, en la visión, o niños con dificultades de aprendizaje. En estos casos, los videojuegos también pueden ser beneficiosos, puesto que permiten desarrollar ciertos aspectos que disminuyen las dificultades de los niños.

Estudios como los de Deutsch, Borbely, Filler, Huhn y Guarrera-Bowlby (2008), nos muestran cómo, utilizando videojuegos (más concretamente "Wii sports"), niños con parálisis cerebral pueden mejorar su movilidad funcional, los procesos perceptivovisuales o el control postural.

Otro aspecto importante está muy relacionado con un aspecto, tratado anteriormente, como es la motivación. El uso de videojuegos que impliquen movimiento (como los de la consola "Wii"), pueden conseguir que, los niños que necesiten algún tipo de rehabilitación realicen durante un mayor tiempo los ejercicios, dado que estarán mucho más motivados que con los ejercicios tradicionales (Ramchandani, Carroll, Buenaventura, Douglas y Liu, 2008).

Se puede decir, además, que los videojuegos son herramientas que facilitan la inclusión de los alumnos con NEE en el aula, convirtiéndose en elementos innovadores, eficaces e inclusivos en el proceso de enseñanza y aprendizaje (Sánchez-Rivas, Ruiz-Palmero y Sánchez-Rodríguez, 2017). Los videojuegos potencian valores y habilidades, además de reforzar el trabajo colaborativo, la actitud positiva ante los errores cometidos y el liderazgo (Earp, Ott, Popescu, Romero y Usart, 2014).

\section{Problema de Investigación}

El siguiente estudio pretende conocer las opiniones y percepciones de los estudiantes del tercer curso del Grado de Educación Primaria, de la Facultad de Educación (Universidad de Extremadura, Badajoz) sobre el uso, las ventajas e inconvenientes que los videojuegos puedan tener como recurso educativo. A colación, nos planteamos las siguientes preguntas de investigación:

a. ¿Los estudiantes del Grado están a favor o en contra del uso de los videojuegos como recurso educativo?

b. ¿Cuáles son los motivos que llevan a los estudiantes del Grado a declinarse por estar a favor o en contra de los videojuegos como recurso educativo?

c. ¿Qué percepción tienen, en relación a la formación de los profesores, sobre el uso de los videojuegos como recurso educativo?

El hecho de realizar estas preguntas o, centrarnos en dicho problema de investigación se debe a la importancia que creemos que los videojuegos pueden tener como recurso educativo. Por ello, consideramos importante conocer si los futuros maestros utilizarán, durante su labor docente, este tipo de recursos o si, por el contrario, son reacios a su utilización.

\section{Metodología}

El presente estudio se desarrolla en el contexto de formación de los alumnos del Grado de Educación Primaria a través de una actividad de reflexión con la finalidad de conocer 
las opiniones que los maestros en formación inicial presentan sobre la utilización de los videojuegos como recurso educativo, permitiéndonos conocer, además, qué saben acerca de dichos recursos. La muestra está constituida por 52 alumnos del tercer curso del Grado de Educación Primaria.

Para recoger los datos, se utilizó el foro, una herramienta disponible en el Moodle del Campus Virtual de la Universidad de Extremadura. Esta herramienta constituye un espacio virtual que permite establecer diálogos entre los participantes de una forma intuitiva y sencilla. En dicho foro planteamos una actividad impulsora que tenía que ser respondida por los alumnos. La actividad fue la siguiente:

- Haz un comentario (según tu opinión) sobre la utilidad o los beneficios que pueden tener los videojuegos en la enseñanza en general y, en particular, de contenidos matemáticos”.

Dicha actividad, al ser abierta, no solo no "contamina" al alumno, si no que permite la reflexión de cada uno sin limitar con caracteres o cercando con temas en concreto.

El hecho de haber utilizado el foro como el "espacio virtual" en el que los alumnos puedan reflexionar, se deben a diversas investigaciones como la de Carvalho, Luengo, Casas y Cubero (2019), en la que nos definen el foro como un espacio que permite, tanto a los alumnos como a los docentes, construir distintos significados mediante la interacción, reflexión conjunta y colaboración entre ellos.

Para realizar la sistematización y el análisis de los datos, seguimos los siguientes pasos: identificar las unidades de información de cada instrumento utilizado para recoger los datos; categorizar dichas unidades; codificar las unidades de información añadiéndolas a su categoría correspondiente; añadir otras categorías libres que surjan en el proceso de codificación y que no las hayamos incluido previamente; analizar las unidades de información.

El procedimiento llevado a cabo para la elaboración del sistema de categorías fue el siguiente: en primer lugar, se realizaron las lecturas de los textos obtenidos y se elaboró un sistema de categorías previo. Posteriormente, se procedió a la validación de dicho sistema de categorías mediante un consenso con otros dos investigadores, quienes categorizaron algunos textos al azar y se obtuvo el coeficiente de Kappa $(\mathrm{k}=0,85)$. Tras el acuerdo de todos los investigadores (haciendo los cambios pertinentes en el sistema), obtuvimos un total de 17 categorías, agrupadas en 5 dimensiones. A cada categoría, además, se le asignó un código que permitiera reconocerlas de una forma simplificada, con el objetivo de facilitar los futuros análisis. Dicho sistema de categorías puede observarse en la tabla 2.

Todo este proceso de análisis, creación de dimensiones, categorización y codificación se realizó a través del Software de Análisis Cualitativo webQDA (Neri, Costa y Moreira, 2011), un programa que nos da muchas facilidades de trabajo dado que, al estar en la nube, podemos acceder desde cualquier lugar. También facilita el trabajo colaborativo, puesto que nos permite agregar usuarios para que colaboren en nuestra investigación (Soto-Ardila, Melo, Caballero y Luengo, 2019). 


\begin{tabular}{|c|c|}
\hline Dimensiones & Categorías \\
\hline A. A favor del uso de los Videojuegos & $\begin{array}{l}\text { A1. Aprendizaje Activo-Participativo. } \\
\text { A2. Constructivismo. } \\
\text { A3. Herramienta Lúdico, motivador, entretenido, } \\
\text { divertido. } \\
\text { A4. Aprendizaje Cooperativo y social. } \\
\text { A5. Potencia la creatividad e imaginación. } \\
\text { A6. Desarrollo de capacidades y habilidades. } \\
\text { A7. Potencia el pensamiento lógico, el razonamiento y la } \\
\text { toma de decisiones. } \\
\text { A8. Buena herramienta de enseñanza. }\end{array}$ \\
\hline B. En contra del uso de los videojuegos & $\begin{array}{l}\text { B1. Efectos psicológicos. } \\
\text { B2. Adicción. } \\
\text { B3. Efectos sociales. } \\
\text { B4. Efectos contra la salud. } \\
\text { B5. Desconocimiento, solo sirve para “jugar”, pérdida de } \\
\quad \text { tiempo. } \\
\text { B6. Videojuegos no adecuados. }\end{array}$ \\
\hline $\begin{array}{l}\text { C. Opiniones Intermedias (Ni a favor, ni en } \\
\text { contra) }\end{array}$ & C1. Opiniones Intermedias. \\
\hline D. Necesidad de formación de los profesores & D1. Necesidad de formación de los profesores \\
\hline
\end{tabular}

Tabla 2 - Sistema de Categorías

\section{Resultados}

En este apartado vamos a explicar los resultados obtenidos en este estudio tratando de responder las preguntas de investigación que hemos detallado previamente.

Esto nos permitirá conocer las opiniones que los alumnos tienen con respecto a este tipo de metodologías, permitiéndonos saber si su utilización puede ser beneficiosa o no, o si puede llegar a ser una herramienta muy utilizada, por ellos, en el futuro (Soto-Ardila, Melo, Caballero y Luengo, 2019).

\section{1. ¿Los estudiantes del Grado están a favor del uso de los videojuegos como recurso educativo o se posicionan en contra del uso de los mismos?}

En relación a esta pregunta de investigación podemos destacar dos dimensiones fundamentales: "a favor del uso de los videojuegos" y "en contra de usarlos”. También

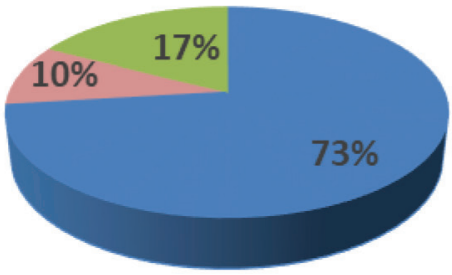

A favor del uso de los videojuegos

En contra del uso de los video juegos

$\mathrm{Ni}$ a favor ni en contra del uso de los videojuegos

Figura 1 - Opiniones de los alumnos sobre el uso de los videojuegos 
podemos añadir una tercera dimensión para englobar a aquellas personas que no se declinan por una dimensión u otra, puesto que les ven el mismo número de ventajas que de inconvenientes. Para estos casos, se añade la dimensión "Ni a favor, ni en contra".

Los datos analizados en relación a esta pregunta de investigación pueden observarse en la siguiente figura:

Como podemos observar, el 73\% de los alumnos, se posicionan a favor de la utilización de los videojuegos como recurso educativo, considerándolos como una herramienta muy útil para enseñar diversos contenidos. Algunos de los ejemplos obtenidos a través del análisis de los textos pueden ser:

- "podemos definir de modo sintético al juego como una actividad voluntaria, fundamentalmente social, en la que se está en relación con otros y en la que se aprenden pautas de comportamiento y valores" (Participante 7).

- "creo que los videojuegos son una herramienta muy útil si son utilizados combinando los componentes lúdicos y didácticos de manera efectiva para el desarrollo de un amplio número de habilidades de los estudiantes" (Participante 51).

- "En él pueden crear mundos a partir de la nada, utilizando conceptos, procedimientos y actitudes vistas en clase, de manera que se fomente el aprendizaje significativo" (Participante 17).

Únicamente encontramos un 10\% de los alumnos de la muestra que se encuentran en contra del uso de los videojuegos como herramienta útil para la enseñanza. Por ejemplo:

- "Existe un alto riesgo de que los niños que están horas y horas sentados delante de la televisión sufran sobrepeso u obesidad debido al sedentarismo" (Participante 42).

- “no le aportan ningún concepto académico sino amplía su vocabulario en otros aspectos. Por lo que es una pérdida de tiempo" (Participante 9).

Por último, resulta interesante destacar que, el 17\% de la muestra se encuentra con opiniones contradictorias, dado que, por un lado, consideran los videojuegos como unas herramientas con un fin positivo en la educación, pero por el otro presenta numerosas desventajas que pueden afectar a la educación y salud del niño. Algún ejemplo de esto es:

- $\quad$ "Los videojuegos como todas las cosas en la vida tienen su lado positivo y su lado negativo. Depende de varios factores como el tiempo que se dedica al juego, el contenido, si este está acorde con la edad de la persona, etc." (Participante 3).

- "En mi opinión los videojuegos son buenos, siempre y cuando sea de manera controlada (temporalmente hablando), ya que se puede crear un vicio que perjudique a estudios, por ejemplo" (Participante 14).

\section{2. ¿Cuáles son los motivos que llevan a los estudiantes del Grado a declinarse por utilizar, o no, los videojuegos como recurso educativo?}

Tras la lectura de todos los textos de la muestra, y tras el posicionamiento de los alumnos en las dimensiones previamente descritas, se analizan los motivos por los cuales se decantan por una dimensión u otra. 
En primer lugar, en relación a cuáles son los motivos por los que los alumnos se encuentran a favor del uso de los videojuegos se pueden destacar las siguientes categorías en consonancia con las indicadas en la figura 2:

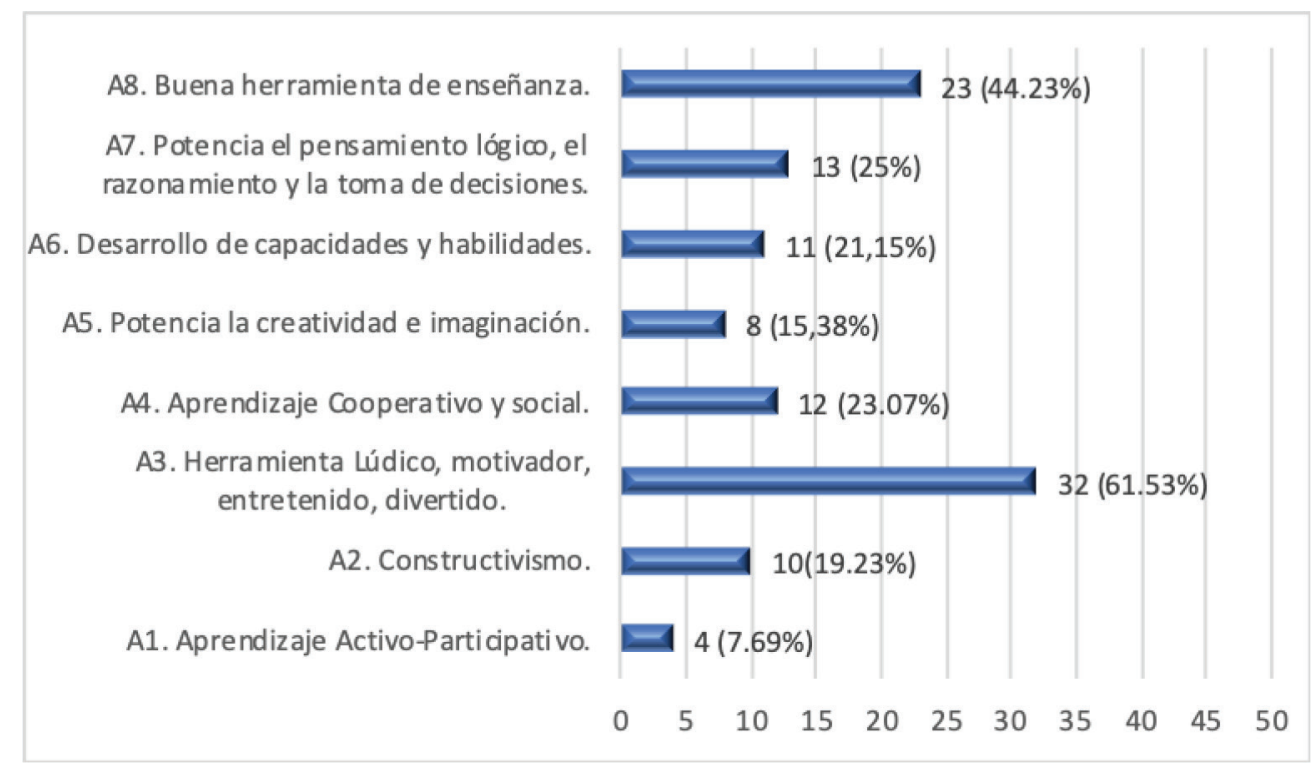

Figura 2 - Motivos a favor del uso de los videojuegos como recurso educativo.

Como se puede observar (en la figura 2), la mayor parte de las personas de la muestra (el 61,53\% de los sujetos) consideran que los videojuegos son una herramienta positiva para los alumnos, puesto que, los niños trabajan de forma lúdica y motivadora (A3), en un ambiente entretenido a la par que divertido. Algunos ejemplos de estos son:

- "Además, creen que aumenta la motivación de los estudiantes, lo que favorece que quieran jugar de forma regular" (Participante 10).

- "El uso de videojuegos para el ámbito educativo puede beneficiar al alumnado ya que aprenderá de una manera más divertida y asimilará mucho mejor los contenidos" (Participante 13).

- "Ayuda a los niños a motivarse, ya que la mayoría de los videojuegos persiguen un objetivo que con ayuda de unas instrucciones puedan llegar a alcanzar, siendo competitivos entre ellos y así motivarse para ganar a sus rivales" (Participante 16).

Otras categorías destacadas por los alumnos de la muestra son: "Potencia el pensamiento lógico, el razonamiento y la toma de decisiones" (A7) y "Buena herramienta de enseñanza” (A8). En la primera, el 25\% de los sujetos coincidían con que los videojuegos, en general, potencian el razonamiento de los niños, mejorando su toma de decisiones y su pensamiento lógico:

- "Debemos destacar también que los juegos potencian el pensamiento creativo y la toma de decisiones en los niños, algo que es esencial de cara a las múltiples 
situaciones a las que se tendrán que enfrentar a lo largo de sus vidas" (Participante 1).

- "En general, los videojuegos ayudan a mejorar la memoria, la lógica, la concentración, el enfoque y la planificación, ya que obligan a los niños a mantener la atención si quieren ganar la partida" (Participante 48).

En el caso de la categoría "Buena herramienta de enseñanza" (44.23\% de la muestra), encontramos las siguientes narraciones:

- "Hoy en día los videojuegos es una de las tendencias de aprendizaje que muchos de los profesores quieren poner en práctica en sus aulas, ya que se pueden trabajar muchos ámbitos y desarrollar múltiples competencias educativa" (Participante 25).

- "En mi opinión, los videojuegos y en general las nuevas tecnologías pueden ayudar bastante a la enseñanza de los niños" (Participante 4).

Dentro de los motivos por los que los sujetos de la muestra se manifiestan en contra del uso de los videojuegos encontramos los siguientes:

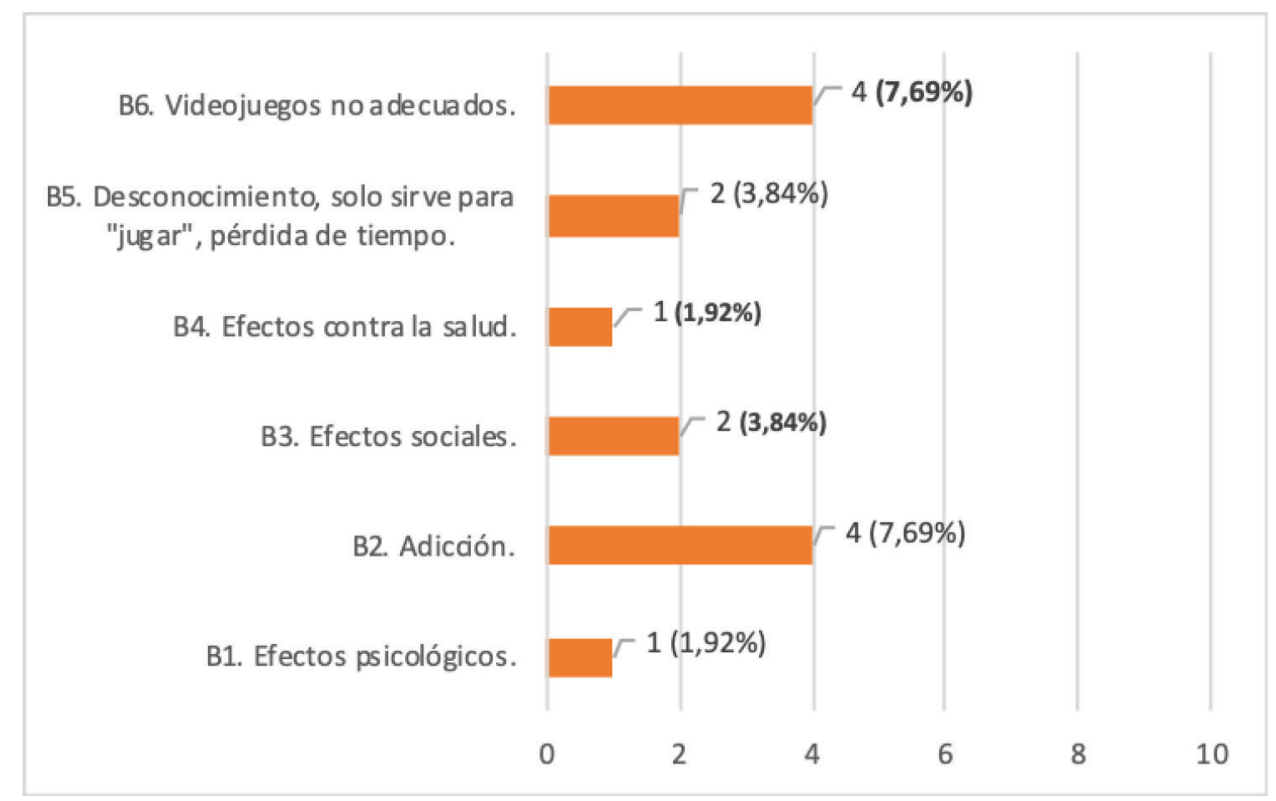

Figura 3 - Motivos en contra del uso de los videojuegos como recurso educativo.

Se pueden destacar dos de los motivos que más participantes mencionaron: Adicción (B2) y Videojuegos no adecuados (B6). En relación a la adicción, el 7,69\% de los sujetos consideraban que los videojuegos podrían crear dependencia al alumno, puesto que, debido a su carácter lúdico, querrían jugar a todas horas, convirtiéndose en un hándicap para ellos. Estos fueron algunos de los relatos: 
- "les dedican tiempo a juegos que no son educativos y que realmente no le aportan ningún tipo de beneficio, al contrario, lo que pueden crear es una adicción" (Participante 9).

- "sus resultados académicos empeoran por dedicar excesivo tiempo a los móviles y los videojuegos" (Participante 50).

Observando la categoría "Videojuegos no adecuados", el 7,69\% de los participantes consideraban que la mayoría de los videojuegos a los que los niños juegan, son de temáticas inadecuadas para ellos, incluyendo contenidos violentos o sensibles que niños de ciertas edades no deberían de conocer, ya que esto podría ocasionarle algunos problemas futuros. Algunos ejemplos son:

- "dejando de lado la formación educativa, ya que la mayor parte de los juegos a los que juegan son de guerra o de matar, conducir, fútbol, es decir, no es nada educativo" (Participante 9).

- $\quad$ "Por lo que resulta muy importante que los padres revisen los juegos a los que acceden, ya que hay muchos juegos cuyos contenidos no son apropiados para los niños" (Participante 48).

\section{3. ¿Qué percepción tienen, en relación a la formación de los profesores, sobre el uso de los videojuegos como recurso educativo?}

Tras la lectura de los relatos, otro de los aspectos observados y que fue destacado en diversas ocasiones, fue la necesidad de formación de los profesores para la utilización de este tipo de herramientas, puesto que, es considerado que, para poder hacer un uso correcto de las mismas, debería de ser necesario estar mucho más formado en el mundo de las nuevas tecnologías y de los videojuegos.

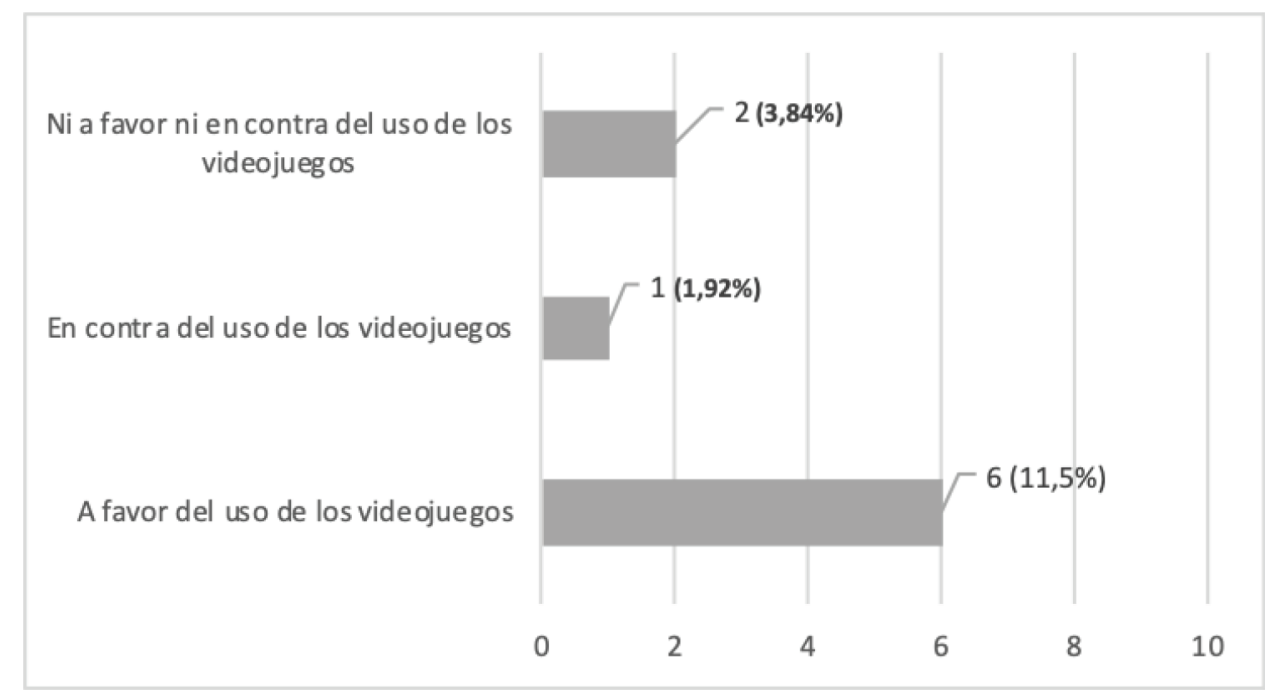

Figura 4 - Necesidad de formación de los profesores en el uso de videojuegos como recurso educativo 
Como se puede observar en el gráfico anterior, independientemente de la dimensión en la que se encuentren, un 17,26\% muestra considera la formación de profesores como algo necesario para utilizar los videojuegos como una herramienta de enseñanza. En este caso podemos ver que, los alumnos que están a favor de emplearlos (el 11,5\% de la muestra), son los que más conciencia tienen de la importancia de una buena formación de los docentes para utilizar este tipo de herramientas.

Algunos de los argumentos empleados por los participantes de la muestra encontramos los siguientes:

- "Para conseguir que los videojuegos cumplan todo lo que he mencionado, creo que los maestros y docentes en general debemos tener una buena formación para que podamos aplicarlos en las aulas en un futuro" (Participante 52).

- "También, requiere una preparación mayor en los docentes, es necesario que estos videojuegos sean elegidos, diseñados o adaptados para ellos, teniendo muy en cuenta lo que se quiera enseñar. Además, deberán hacer una evaluación para ver y valorar si el uso de esta herramienta está funcionando correctamente “. (Participante 33).

\section{Discusión y Conclusiones}

Una vez revisada la bibliografía, analizados todos los datos y extraídos los resultados, pasamos a la discusión y conclusiones del estudio. Entre las conclusiones que podemos obtener de nuestra investigación, mencionamos las siguientes:

Se puede comprobar que la mayor parte de los sujetos participantes se muestran a favor de utilizar esta metodología (los videojuegos) como recurso didáctico, dado que afirman que los videojuegos presentan numerosos beneficios para el desarrollo del alumno. Entre ellos, consideran que la motivación es uno de los factores más importantes, dado que los videojuegos, al ser elementos lúdicos, entretienen, motivan y captan la atención de los alumnos, en concordancia con los hallazgos de autores como Rosas, Nussbaum, Cumsille, Marianov, Correa y Flores (2003) o Brustolin y Malta (2017).

Otro de los motivos en los que la mayor parte de la muestra coincidía era en el desarrollo del pensamiento lógico, crítico, y la resolución de problemas, que se ve beneficiada al utilizar videojuegos, coincidiendo con lo que dice Kirriemuir y Mcfarlane (2004) y Higgins (2001).

Si tenemos en cuenta a los maestros en formación inicial que se mostraban en contra de utilizar los videojuegos como recursos para enseñar, se puede decir que, algunos de los motivos que exponían estaban relacionados con el uso de videojuegos inadecuados ya que pueden llegar a influir negativamente en el niño, llegando a ocasionar problemas como la agresividad, violencia, o el aislamiento del jugador (Ferguson, 2010).

Otro de los motivos, a los que hacían referencia estos autores, era la adicción, dado que consideraban que el niño puede dejar de prestar atención a otras actividades por pasar demasiado tiempo jugando, e incluso dejar de relacionarse con sus iguales ocasionando aislamiento. A pesar de ello, en la literatura científica, se ha podido comprobar que esas opiniones no son compartidas por todos los autores, como es el caso de Padilla, Collazos, 
Gutiérrez y Medina (2012), Colwel, Grady y Rhaiti (1995) o Estallo (1995). Estos autores consideran que, una vez que al niño deja de llamarle la atención un videojuego al conocer sus mecánicas, poco a poco deja de jugar con él, alejándose el riesgo de adicción. Por otra parte, señalan que el videojuego, además, hace más sociable al jugador.

Finalmente, cabe destacar la importancia que tiene el hecho de utilizar este tipo de metodología (basada en el estudio cualitativo de los datos), la cual nos permite profundizar mucho más en aquellos aspectos que se pretenden obtener tras la utilización de la actividad (o pregunta) impulsora, consiguiendo una mayor cantidad de datos para el estudio y unos resultados más enriquecedores.

\section{Agradecimientos.}

Este trabajo ha sido financiado por el Ministerio de Educación y Formación Profesional (contrato FPU, código FPU17/00897) y por la Junta de Extremadura (Ayuda a grupos TIC016, expediente GR15061).

\section{Referencias}

Aguilar Gutiérrez, E., Rubio Florido, I., \& Viñals Blanco, A. (2013). El ocio digital como recurso para el aprendizaje, la socialización y la generación de capital social. Revista de la Asociación de Sociología de la Educación, 6(2), 196-209.

Albarracín, L., Hernández, A., \& Gorgorió, N. (2019). Razonamiento tridimensional promovido por un videojuego. Uno - Revista de Didáctica de las Matemáticas, $83(1), 62-67$.

Alonso, L., \& Yuste, R. (2015). Teorías de la educación de adultos que subyacen en el uso de videojuegos. Education in the Knowledge Society, 15(4), 160-183.

Brustolin, F. J., \& Malta, J. E. (2017). Análise de Gamificaçao no Simulador de Operaçoes Cibernéticas (SIMOC). RISTI, Revista Ibérica de Sistemas y Tecnologías de Información, 23(1), 103-118. DOI: 10.17013/risti.23.103-118

Carvalho, J., Luengo, R., Casas, L., \& Cubero, J. (2019). What is better to study, The Printed Book or the Digital Book?: An Exploratory Study of Qualitative Nature. In A. Costa, \& A. Moreira (Ed.), Computer Supported qualitative Research. New trends on Qualitative Research (pp 34-45). Switzerland: Springer.

Colwell, J., Grady, C., \& Rhaiti, S. (1995). Computer Games, selfEsteem and Gratification of Needs in Adolescents. Journal of Community and Applied Social Psychology, 5(3), 195-206.

Del Moral, M., Guzmán, A., \& Fernández, L. (2014). Serious Games: escenarios lúdicos para el desarrollo de las inteligencias múltiples en escolares de primaria. Edutec, 47, 37-51. Doi: https://doi.org/10.21556/edutec.2014.47.121

Deutsch, J. E., Borbely, M., Filler, J., Huhn, K., \& Guarrera-Bowlby, P. (2008). Use of a low-cost, commercially available gaming console (wii) for rehabilitation of an adolescent with cerebral palsy. Physical Therapy, 88(10), 1196-1207. 
Dondi, C., Edvinsson, B., \& Moretti, M. (2004). Why choose a game for improving learning and teaching processes? In: Pivec, M., Koubek, A., \& Dondi, C. (Ed.), Guidelines for game-based learning (pp. 20-76). Lengerich: Pabst Science Publ.

Earp, J., Ott, M., Popescu, M., Romero, M., \& Usart, M. (2014). Supporting human capital development with serious games: An analysis of three experiences. Computers in Human Behaviour, 30, 715-720. Doi: https://doi.org/10.1016/j.chb.2013.09.004

Eguía, J. L., Contreras-Espinosa, R., \& Solano-Albajes, L. (2013). Videojuegos: conceptos, historia y su potencial como herramientas para la educación. Revista 3 Ciencias $T I C, 1(2), 1-14$. Recuperado en 01 de marzo de 2019 de https://www.3ciencias. com/articulos/articulo/videojuegos-conceptoshistoria-y-su-potencial-comoherramienta-para-la-educacion/

Estallo, J. A. (1995): Los videojuegos: juicios y pre-juicios. Barcelona: Planeta.

Ferguson C. J., (2010). Blazing angels or resident evil? Can violent video games be a force for good?. Review of General Psychology, 14(2), 68-81.

Frasca, G. (2001). Videogames of the oppressed: Videogames as a means for critical thinking and debate (Tesis Doctoral). Escuela de Literatura, Comunicación y Cultura, Georgia.

Higgins, S. (2001). ICT and teaching for understanding. Evaluation and Research in Educaction, 15(3), 164-171.

Kirriemuir, J. \& Mcfarlane, A. (2004) Literature review in games and Learning. Bristol: Futurelab Series Report.

Martinez-Piñeiro, E., Vila, E., \& Gewerc, A. (2018). El papel de la familia en la construcción de la competencia digital. RISTI, Revista Ibérica de Sistemas y Tecnologías de Información, 28(1), 1-13. DOI: 10.17013/risti.28.1-13

Muros, B., Aragón, Y., \& Bustos, A. (2013). La ocupación del tiempo libre de jóvenes en el uso de videojuegos y redes. Comunicar, 2O(40), 31-39. DOI: https://doi. org/10.3916/C40-2013-02-03

Neri, F., Costa, A. P., \& Moreira, A. (2011). Análise de Dados Qualitativos Suportada pelo Software webQDA. In Atas da VII Conferência Internacional de TIC na Educação: Perspetivas de Inovação (CHALLANGES2011), 49-56.

Padilla, N., Collazos, C. A., Gutiérrez, F. L., \& Medina, N. (2012). Videojuegos educativos: teorías y propuestas para el aprendizaje en grupo. Ciencia e ingeniería neogranadina, 22(1), 139-150.

Ramchandani, A., Carroll, K., Buenaventura, R., Douglas, J., \& Liu, J. (2008). Wiihabilitation increases participation in therapy. In J. Fung (Presidency). Virtual Rehabilitation 2008 Conferencia. Conferencia llevada a cabo en el congreso. Vancouver, Canada. DOI: 10.1109/ICVR.2008.4625132

Rosas, R., Nussbaum, M., Cumsille, P., Marianov, V., Correa, M., \& Flores, P. (2003). Beyond Nintendo: Design and assessment of educational video games for first and second grade students. Computers and Education, 4O(1), 71-94. 
Sánchez-Rivas, E., Ruiz-Palmero, J., \& Sánchez-Rodríguez, J. (2017). Videojuegos frente a fichas impresas en la intervención didáctica con alumnado con necesidades educativas especiales. Educar, 53(1), 29-48. DOI: https://doi.org/10.5565/rev/ educar.844

Soto-Ardila, L. M. (2015). Estudio sobre la aplicación de los videojuegos en la enseñanza de la matemática: elaboración de un prototipo de juego (Trabajo de fin de Máster). Universidad de Extremadura, Badajoz.

Soto- Ardila, L. M., Melo, L., Caballero, A., \& Luengo, R. (2019). Análisis cualitativo de las opiniones de los estudiantes del Grado de Educación Primaria sobre el uso de los videojuegos como recurso educativo. En Congreso Ibero-americano en Investigación Cualitativa (pp.169-178). Lisboa, Portugal.

Torres-Toukoumidis, A., Romero-Rodríguez, L., Pérez-Rodriguez, M. A., \& Björl, S. (2016). Desarrollo de habilidades de lectura a través de los videojuegos: Estado del arte. OCNOS - Revista de Estudios sobre Lectura, 15(2), 37-49. DOI: 10.18239/ ocnos_2016.15.2.1124

Zyda, M. (2005). From visual simulation to virtual reality to games. Computer, 38(9), 25-32. 
